\title{
Cannabis and Psychosis: Are We any Closer to Understanding the Relationship?
}

\author{
Ian Hamilton $^{1} \cdot$ Mark Monaghan $^{2}$ \\ Published online: 4 June 2019 \\ (C) The Author(s) 2019
}

\begin{abstract}
Purpose of Review This paper provides an update from the literature on understanding of the relationship between cannabis and schizophrenia. In particular, the paper focuses on the latest findings and remaining areas that require investigation.

Recent Findings Three hypotheses have emerged as potential explanations for the association between cannabis and schizophrenia, namely cannabis can trigger schizophrenia, cannabis is used to mitigate symptoms of schizophrenia, and there are common factors which might account for the association. Biological and genetic factors dominate this field of research; this has been at the expense of exploring social and cultural contributory factors which influence cannabis and schizophrenia.

Summary The evidence for cannabis acting as a causal factor for schizophrenia has so far not been established. Research needs to extend beyond males drawn from western countries if we are to advance knowledge and understanding of the link between cannabis use and schizophrenia.
\end{abstract}

Keywords Cannabis · Psychosis · Schizophrenia $\cdot$ Diagnosis · Cannabis use disorder $\cdot$ Sex

\section{Introduction}

An estimated 192 million people used cannabis in 2016 demonstrating its status as the most popular drug after alcohol and tobacco globally [1]. As with all drugs, there are risks associated with its use; some risk is due to the substance and some will depend on the regulatory status of the drug in the local jurisdiction that the individual resides in. One risk of using cannabis continues to attract significant attention, namely the risk of developing psychosis. Distinguishing between causation and correlation on this issue has proved to be a challenge. However, it is critical that we understand how risks such as developing psychosis and schizophrenia for those that are exposed to cannabis unfold, so this knowledge could be used to

This article is part of the Topical Collection on Schizophrenia and Other Psychotic Disorders

Ian Hamilton

ian.hamilton@york.ac.uk

1 Health Sciences, University of York, York, UK

2 Social Policy, University of Birmingham, Birmingham, UK reduce harm and to more effectively target scarce public health resources to high risk groups.

Scholars and clinicians have been aware of a link between cannabis and schizophrenia for over a century [2]. However, it wasn't until cannabis use became popular in western countries during the 1960's that researchers began to investigate the nature of the relationship. The seminal observational study by Andreasson and colleagues set the research agenda on this topic, reporting that cannabis was an independent risk factor for schizophrenia with $6 \%$ of heavy cannabis users going on to develop schizophrenia [3].

Three hypotheses outline the potential relationship between cannabis and schizophrenia in contemporary literature:

1. Cannabis can trigger schizophrenia in an individual who would not have developed the illness if they had not been exposed to the drug.

2. Individuals with a predisposition to schizophrenia use cannabis to mitigate the prodromal symptoms of schizophrenia, referred to as reverse causation.

3. Common cause suggests that other factors are responsible for the relationship such as childhood trauma or genetics for example. 
Although the first of these hypotheses appears to be simple, it has proved to be difficult to investigate with confidence [4]. Research relies on observational methods mainly with all the limitations these have, but there are other problems in exploring this theory. It is widely thought that the dose of cannabis is a factor in increasing the risk of psychosis [5], but there is significant heterogeneity in the assessment of dose. Some researchers use exposure of 50 times or more, others use the proxy of last month usage to indicate regular use as opposed to infrequent or occasional use of the drug [6•]. This is compounded by the diagnostic criteria for cannabis-induced psychosis that rely on subjective assessment [7]. For instance, an assessing clinician or researcher trying to establish that cannabis played a causal role for an individual presenting with psychosis would need to decide how recent use of cannabis should be in order to make this differential diagnosis. The possibility for error is amplified when considering that metabolites of cannabis can be detected for weeks following exposure [8], clearly raising the risk of a false positive association and diagnosis of cannabis-induced psychosis.

The second hypothesis suggests individuals who are predisposed to schizophrenia are drawn to cannabis to mitigate the early signs of negative symptoms of schizophrenia. Although plausible, it should be stressed that several studies show how this is not the main reason these individuals consistently give as their motivation to use cannabis. Pleasure and recreation are the main reasons given by patients [9-11]. Although these reasons are clearly subjective and based on individual perception, they are important to understand particularly in the clinical setting as they provide an insight to the patients' motivation for using cannabis even if this use is detrimental to their mental health.

An interesting and promising avenue of research is the potential that cannabis or rather the role a group of compounds found in cannabis might offer in treating schizophrenia. Cannabidiols have been shown to provide therapeutic value in the treatment of schizophrenia with a relatively low risk of adverse effects [12]. This might add credibility to the second hypothesis as individuals could be benefiting from using cannabis that contains these compounds. Mitigating factors here, however, include the emerging evidence that the composition of cannabis in recent years has been changing $[12,13 \cdot 14]$. These changes revolve around the ratios of $\Delta^{9}$-tetrahydrocannabinol (THC) and cannabidiols (CBD), some of the more prominent compounds in cannabis. Higher potency strains are higher in THC and lower in CBD.

This aspect is rarely assessed; even when potency or strength is investigated, the categories used to describe this factor are inconsistent and sometimes too generic to allow replication or translation. The increasing potency of cannabis that is available is thought to play a role in the risk of developing psychosis and other cannabis use disorders $[15,16]$. This leads onto a further problem namely the qualifying period of experiencing psychosis that is required to qualify for a diagnosis of cannabis-induced psychosis. Some people will have a short-lived acute reaction to cannabis while others will go on to develop a longer-term problem. This offers the potential of over counting or under counting cases depending on where the individual presents to, how long they are assessed for and the depth and quality of the assessment conducted at that point in time.

The third hypothesis which proposes a common cause such as a shared genetic liability is responsible for the link between cannabis and schizophrenia [17, 18]. This has been explored more recently using sophisticated methods of analysis to try and untangle the relationship between cannabis and schizophrenia. One example used Mendelian randomisation to investigate the direction of the relationship in a study using a genome database [18]. This reported that there is a slightly increased risk of developing schizophrenia following initiation of cannabis with stronger evidence that schizophrenia predicts cannabis initiation.

So far, genetic orientated research has failed to provide a plausible explanation involving a purely biological or genetic factor that makes clear the direction of the relationship between cannabis and schizophrenia. Although it is possible that there is a relationship and even if that were to emerge as causal, it would be influenced significantly by the individual's environment [19]. Most research in this field relies on animal studies which have their own limitations, but they still have a contribution to theoretical modelling.

\section{Knowledge Gaps}

Beyond the aforementioned limitations, there are some other gaps in research and understanding of the relationship between cannabis and schizophrenia. The Sex and Gender Equity in Research (SAGER) guidelines acknowledge the inattention given to sex generally [20]. This appears to have limited our understanding of the links between cannabis and schizophrenia for women. The study by Andréasson included an all-male sample; research since then has either oversampled males or failed to report differences by sex [21•]. Reporting sex in studies exploring cannabis and schizophrenia would not only improve our knowledge of the impact this has on females but may also provide useful information and insights for men [22]. If we had more information about the differences between the sexes in the risk of developing cannabis psychosis, this could prove useful in adding to knowledge about risk factors and who would benefit from targeted public health interventions to minimise the risk [23••].

Research into cannabis psychosis originates and continues to be dominated by participants drawn from western countries. Cannabis is used in most countries across the world although again we know more about use of the drug among western 
populations than any other. A recent review of the Indian literature investigating psychiatric problems including psychosis associated with cannabis use found a limited focus on aetiology and that all the studies bar one had all male samples [24]. This clearly leaves us with a skewed view of the impact on health resulting from use of cannabis. Drug use and its consequences are known to be sensitive to cultural influences; studies on cannabis psychosis have been dominated by samples drawn from America, Australia, and Europe. In some parts of Asia, there appears to be lower population use of cannabis which might in part account for the lower incidence of psychosis found in these populations [25].

Cannabis and tobacco are frequently used in combination so attention has turned to the synergistic effects of cannabis and tobacco on psychosis [26, 27]. Tobacco appears to be an independent risk factor for psychosis. As rates of cigarette smoking are elevated in those with psychosis compared with the general population there are some obvious general health benefits if cigarette use were to be reduced in this population [28]. As there are few modifiable risk factors for schizophrenia, there is a good case for trying to decouple tobacco from cannabis in those cannabis using populations as this could reduce the risk of psychosis by itself.

It is sometimes difficult for individuals to distinguish between cannabis and tobacco dependence particularly when they are used in combination. So even when someone has psychosis thought to have been associated with cannabis use, they can struggle to abstain from cannabis [29•]. Little evidence exists as to how this group can be encouraged and supported to abstain from cannabis use with the aim of reducing the impact of psychosis on their lives. There is the opportunity to learn from those with psychosis who have successfully reduced or abstained completely from using cannabis $[30,31]$.

\section{Conclusion}

We need to carefully balance enquiry into cannabis and schizophrenia between neurophysiology and the social aspects of cannabis psychosis. Attention and research funding leans towards the biological hypothesis at the expense of the cultural $[32 \bullet \cdot]$. As cannabis use and the development of psychosis are both influenced by social as well as biological factors, it is important that we keep pursuing a balanced blend of enquiry.

Dose and frequency of cannabis use continue to be reliable indicators in the risk of developing psychosis [33]. However, some individuals appear to be sensitive to low levels of exposure to cannabis; it is still not clear whether that is due to increasing potency of cannabis or some other factor such as genetics.
Overall, we still have insufficient information and knowledge about who is at risk of developing cannabis psychosis prior to cannabis exposure to reliably produce a public health prevention strategy. As an increasing number of American states allow access to cannabis for recreational or medicinal purposes, this presents a large naturalistic experiment involving these populations, it will be some years before we can judge the impact of these regulatory changes on health and incidence and prevalence of psychosis in particular [34].

\section{Compliance with Ethical Standards}

Conflict of Interest Ian Hamilton and Mark Monaghan each declare no potential conflicts of interest.

Human and Animal Rights and Informed Consent This article does not contain any studies with human or animal subjects performed by any of the authors.

Open Access This article is distributed under the terms of the Creative Commons Attribution 4.0 International License (http:// creativecommons.org/licenses/by/4.0/), which permits unrestricted use, distribution, and reproduction in any medium, provided you give appropriate credit to the original author(s) and the source, provide a link to the Creative Commons license, and indicate if changes were made.

\section{References}

Papers of particular interest, published recently, have been highlighted as:

- Of importance

•- Of major importance

1. United Nations Office on drugs and Crime (2018) Analysis of drug markets, opiates, cocaine, cannabis, synthetic drugs. https://www. unodc.org/wdr2018/prelaunch/WDR18_Booklet_3_DRUG MARKETS.pdf. Accessed 20/3/19.

2. Mills JH. Cannabis Britannica: empire, trade and prohibition 1800 1928. Oxford: Oxford University Press; 2003.

3. Andréasson S, Engström A, Allebeck P, Rydberg U. Cannabis and schizophrenia a longitudinal study of Swedish conscripts. Lancet. 1987;330(8574):1483-6.

4. Hall W, Degenhardt L. Is there a specific 'cannabis psychosis'. Marijuana and madness: psychiatry and neurobiology. Cambridge: Cambridge University Press; 2004. p. 89-100.

5. Marconi A, Di Forti M, Lewis CM, Murray RM, Vassos E. Metaanalysis of the association between the level of cannabis use and risk of psychosis. Schizophr Bull. 2016;42(5):1262-9.

6. Curran HV, Hindocha C, Morgan CJ, Shaban N, Das RK, Freeman TP. Which biological and self-report measures of cannabis use predict cannabis dependency and acute psychotic-like effects? Psychol Med. 2018;4:1-7 This paper makes the case for combining selfreported cannabis use with biological testing as way of improving measurement.

7. Hall W. What has research over the past two decades revealed about the adverse health effects of recreational cannabis use? Addiction. 2015;110(1):19-35. 
8. Taylor M, Lees R, Henderson G, Lingford-Hughes A, Macleod J, Sullivan J, et al. Comparison of cannabinoids in hair with selfreported cannabis consumption in heavy, light and non-cannabis users. Drug Alcohol Rev. 2017;36(2):220-6.

9. Schofield D, Tennant C, Nash L, Degenhardt L, Cornish A, Hobbs $\mathrm{C}$, et al. Reasons for cannabis use in psychosis. Aust N Z J Psychiatry. 2006;40(6-7):570-4.

10. Dekker N, Linszen DH, De Haan L. Reasons for cannabis use and effects of cannabis use as reported by patients with psychotic disorders. Psychopathology. 2009;42:350-60.

11. Hamilton I. Cannabis, psychosis and schizophrenia: unravelling a complex interaction. Addiction. 2017;112(9):1653-7.

12. ElSohly MA, Mehmedic Z, Foster S, Gon C, Chandra S, Church JC. Changes in cannabis potency over the last 2 decades (19952014): analysis of current data in the United States. Biol Psychiatry. 2016;79(7):613-9.

13. Chandra S, Radwan MM, Majumdar CG, Church JC, Freeman TP, ElSohly MA. New trends in cannabis potency in USA and Europe during the last decade (2008-2017). Eur Arch Psychiatry Clin Neurosci. 2019;269(1):5-15 This study provides insights into the way cannabis potency has increased across several countries, this is important as cannabis potency is believed to increase the risk of problems such as psychosis.

14. Dujourdy L, Besacier F. A study of cannabis potency in France over a 25 years period (1992-2016). Forensic Sci Int. 2017;272:72-80.

15. Arterberry BJ, Padovano HT, Foster KT, Zucker RA, Hicks BM. Higher average potency across the United States is associated with progression to first cannabis use disorder symptom. Drug Alcohol Depend. 2019;195:186-92.

16. Di Forti M, Quattrone D, Freeman T, Tripoli G, Gayer-Anderson C, Rodriguez $\mathrm{V}$, et al. The contribution of cannabis use to variation in the incidence of psychotic disorder across Europe (EU-GEI): a multicentre case-control study. Lancet Psychiatry. 2019;6(5):42736 online first.

17. Verweij KJ, Abdellaoui A, Nivard MG, Cort AS, Ligthart L, Draisma HH, et al. Genetic association between schizophrenia and cannabis use. Drug Alcohol Depend. 2017;(171):117-21.

18. Colizzi M, Weltens N, McGuire P, Lythgoe D, Williams S, Van Oudenhove L, et al. Delta-9-tetrahydrocannabinol increases striatal glutamate levels in healthy individuals: implications for psychosis. Mol Psychiatry. 2019. https://doi.org/10.1038/s41380-019-0374-8.

19. Karl T, Arnold JC. The interactive nature of cannabis and schizophrenia risk genes. In: Handbook of cannabis and related pathologies. Cambridge: Academic; 2017. p. 335-44.

20. Heidari S, Babor TF, De Castro P, Tort S, Curno M. Sex and gender equity in research: rationale for the SAGER guidelines and recommended use. Research Integrity and Peer Review. 2016;1(1):2.

21. Del Boca FK. Addressing sex and gender inequities in scientific research and publishing. Addiction. 2016;111(8):1323-5 This was an important editorial which drew attention to the need to include female participants in research and report findings.

22. Hamilton I, Galdas P, Essex H. Cannabis psychosis, gender matters. Adv Dual Diagn. 2015;8(3):153-62.
23.• Crocker CE, Tibbo PG. The interaction of gender and cannabis in early phase psychosis. Schizophr Res. 2018;194:18-25 This paper makes a compelling case for exploring the impact of cannabis on females and how this might help us understand the risk factors.

24. Singh S, Balhara YP. A review of Indian research on co-occurring cannabis use disorders \& psychiatric disorders. Indian J Med Res. 2017;146(2):186-95.

25. Lee TY, Kwon JS. Psychosis research in Asia: advantage from low prevalence of cannabis use. NPJ Schizophr. 2016;2:1.

26. Gurillo P, Jauhar S, Murray RM, MacCabe JH. Does tobacco use cause psychosis? Systematic review and meta-analysis. Lancet Psychiatry. 2015;2(8):718-25.

27. Colizzi M, Murray R. Cannabis and psychosis: what do we know and what should we do? Br J Psychiatry. 2018;212(4):195-6.

28. Gage SH, Munafò MR. Smoking as a causal risk factor for schizophrenia. Lancet Psychiatry. 2015;2(9):778-9.

29. Murray RM, Di Forti M. Cannabis and psychosis: what degree of proof do we require? Biol Psychiatry. 2016;79(7):514-5 This paper takes stock of the evidence and challenges us to think about how we should communicate the risks associated with cannabis.

30. Rebgetz S, Hides L, Kavanagh DJ, Choudhary A. Natural recovery from cannabis use in people with psychosis: a qualitative study. J Dual Diagn. 2015;11(3-4):179-83.

31. Berry K, Palmer T, Gregg L, Barrowclough C, Lobban F. Attachment and therapeutic alliance in psychological therapy for people with recent onset psychosis who use cannabis. Clin Psychol Psychother. 2018;25(3):440-5.

32.• Karcher NR, Barch DM, Demers CH, Baranger DA, Heath AC, Lynskey MT, et al. Genetic predisposition vs individual-specific processes in the association between psychotic-like experiences and cannabis use. JAMA Psychiatry. 2019;76(1):87-94 This paper makes clear that while genetic factors appear to play a role in the relationship between cannabis and psychosis individual environmental factors are important but less well understood.

33. Murray RM, Quigley H, Quattrone D, Englund A, Di Forti M. Traditional marijuana, high-potency cannabis and synthetic cannabinoids: increasing risk for psychosis. World Psychiatry. 2016;15(3):195-204.

34. Di Forti M, Quattrone D, Tripoli G, La Cascia C, Ferraro L, GayerAnderson C, et al. O12. 4. Some of the individual differences in risk to develop psychosis among cannabis users can be explained by where they live and by their age at first use. Schizophr Bull. 2018;44(Suppl 1):S110.

Publisher's Note Springer Nature remains neutral with regard to jurisdictional claims in published maps and institutional affiliations. 